\title{
Computer assisted diagnosis of ovarian cancer in primary care
}

\author{
Moving closer, but still some way off
}

\author{
William Hamilton professor of primary care diagnostics
}

Peninsula College of Medicine and Dentistry, Exeter EX2 4SG, UK

\begin{abstract}
More than 6500 women are diagnosed with ovarian cancer each year in the United Kingdom. This cancer has a relatively poor prognosis-five year survival is $41 \%$. $^{1}$ Screening is not available, although a large UK trial is due to report in 2015, so this may change. ${ }^{2}$ Currently, the diagnosis is made almost entirely as a result of women reporting symptoms to primary care. ${ }^{3}$ However, several factors make the diagnosis of ovarian cancer difficult. The cancer is relatively rare-full time general practitioners (GPs) will encounter it once every five years on average, so they build up little personal experience of diagnosing the disease. Many of the symptoms, such as urinary frequency or abdominal pain, are non-specific, with causes other than ovarian cancer more likely. ${ }^{4}$ Furthermore, women are not as knowledgeable about the symptoms of ovarian cancer as they are about other cancers. ${ }^{5}$ This encourages late presentation, with women unlikely to mention the possibility of ovarian cancer in the consultation. Contrast this with breast cancer, where most women finding a breast lump will report it swiftly and will expect the possibility of cancer to be discussed at the consultation.
\end{abstract}

In the linked study (doi:10.1136/bmj.d8009), Hippisley-Cox and Coupland look at a different aspect of the diagnosis of ovarian cancer- that of computer assisted diagnosis. ${ }^{6}$ They identified the symptoms of ovarian cancer in a large electronic database, created a predictive algorithm, and then tested it in a different subset of the database. The algorithm performed well, with an area under the receiver operating characteristic curve of $84 \%$. The authors hope that their algorithm could be used routinely to identify women at high risk of harbouring ovarian cancer who could then be offered testing. How realistic is this?

There are several stages before computerised support for a diagnosis of cancer can become a reality. Creation of an algorithm is generally the first one-they have been created for several other cancers, such as colorectal cancer. ${ }^{7}$ Ideally, such algorithms should be validated in a different dataset from the one in which they were created because their performance is generally worse when tested in a second environment. Some of this poorer performance reflects coding idiosyncrasies peculiar to each dataset. Several computer and coding systems are in use in UK general practice, with the Hippisley-Cox algorithm derived from one of the most common ones. However, primary care record keeping is far from standardised, especially for symptoms. Variation between GPs in recording styles would have a considerable impact on the algorithm. For example, loss of appetite was recorded in $0.5 \%$ of patients in both the derivation and validation cohorts yet was recorded in $1.5 \%$ of controls in a similar study that examined both written and computerised records. ${ }^{4}$ This difference probably reflects "hidden" information that cannot be extracted by simple computer searches. ${ }^{8}$ A change of this size may invalidate the algorithm. Furthermore, GPs do not record every symptom that is mentioned in the consultation-much of the time they document only diagnoses and treatment. Once GPs knew that their records of symptoms were being incorporated into an algorithm, their style of record keeping would probably change, again weakening the current algorithm, although it could be updated.

We also need to know if these algorithms can easily be integrated into general practice software. An early attempt put several computers out of action in a practice in Sheffield. ${ }^{9}$ Various teams are working on incorporating diagnostic software into practice computers, so this aspect can probably be solved satisfactorily, for the popular general practice computer systems at least. There is also good evidence that GPs are willing to use algorithms to improve clinical care. Cardiovascular risk assessment is standard practice in primary care. GPs have also shown that they are willing to use scoring systems to identify possible colorectal cancer. ${ }^{10}$ Several hundred English GPs are currently piloting a paper based risk scoring system aimed at identifying lung or colorectal cancers that would not qualify for urgent investigation under National Institute for Health and Clinical Excellence (NICE) recommendations. ${ }^{11}$

Once these hurdles are overcome, it will be important to select an "action level" for patients identified by any cancer diagnostic algorithm. False positives-patients in whom the algorithm identifies possible cancer but who do not have the disease-will be common. In cancer diagnostics, the action level with the highest possible sensitivity is usually selected to ensure as few 
cancers as possible are missed. The upper limit is then set when the false positive rate becomes unacceptably high. The disadvantages of false positive results can be minimised by having a simple "second level" test that can refine the risk further before undertaking more invasive tests. This is particularly important if an algorithm is used away from the consultation room. For instance, GPs could run regular sweeps of their electronic records for patients with symptom patterns that might represent cancer and invite patients who test "positive" for additional testing.

Despite its rarity, ovarian cancer may be a sensible choice for field testing an algorithm. Measurement of $\mathrm{Ca} 125$ is now thought to be useful in primary care, although it too has false positives and false negatives. ${ }^{12}$ Furthermore, GPs in England now have improved access to transvaginal ultrasound, which provides a reasonably rapid mechanism for confirming or rebutting the diagnosis.

Hippisley-Cox and Coupland's study has taken us one step closer to computer assisted diagnosis of cancer, although several steps remain. One final step must take place outside the GP's surgery. All systems that aim to improve identification of cancer in primary care will lead to increased demand for definitive tests. There is no point in increasing public awareness, as with recent TV campaigns for bowel cancer, or increasing the willingness of GPs to investigate, unless additional investigative services can be provided-and paid for.

Competing interests: The author has completed the ICMJE uniform disclosure form at www.icmje.org/coi_disclosure.pdf (available on request from the corresponding author) and declares: no support from any organisation for the submitted work; no financial relationships with any organisations that might have an interest in the submitted work in the previous three years; no other relationships or activities that could appear to have influenced the submitted work.

Provenance and peer review: Commissioned; not externally peer reviewed.

1 Office for National Statistics. Statistical bulletin: Cancer incidence and mortality in the UK, 2006-2008. 2011.

2 Menon U, Gentry-Maharaj A, Hallett R, Ryan A, Burnell M, Sharma A, et al. Sensitivity and specificity of multimodal and ultrasound screening for ovarian cancer, and stage distribution of detected cancers: results of the prevalence screen of the UK Collaborative Trial of Ovarian Cancer Screening (UKCTOCS). Lancet Oncol 2009;10:327-40.

3 Bankhead C, Kehoe S, Austoker J. Symptoms associated with diagnosis of ovarian cancer: a systematic review. BJOG 2005;112:857-65.

4 Hamilton W, Peters TJ, Bankhead C, Sharp D. Risk of ovarian cancer in women with symptoms in primary care: population based case-control study. BMJ 2009:339:b2998.

5 Target Ovarian Cancer. Pathfinder Study. London: Target Ovarian Cancer, 2009:32.

6 Hippisley-Cox J, Coupland C. Identifying women with suspected ovarian cancer in primary care: derivation and validation of algorithm. BMJ 2012;344:d8009

7 Marshall T, Lancashire R, Sharp D, Peters T, Cheng K, Hamilton W. The Diagnostic Performance of Scoring systems to identify symptomatic colorectal cancer compared to current referral guidance. Gut 2011;60:1242-8.

8 Tate AR, Martin AGR, Ali A, Cassell JA. Using free text information to explore how and when GPs code a diagnosis of ovarian cancer: an observational study using primary care records of patients with ovarian cancer. BMJ Open 2011;1(1):e000025.

9 Jiwa M, Skinner P, Coker A, Shaw L, Campbell M, Thompson J. Implementing referral guidelines: lessons from a negative outcome cluster randomised factorial trial in general practice. BMC Fam Pract 2006;7:65.

10 Khan N; NCRI Colorectal Clinical Studies Group. Implementation of a diagnostic tool for symptomatic colorectal cancer in primary care: a feasibility study. Primary Health Care Res Dev 2009;10:54-64.

11 National Institute for Health and Clinical Excellence. Referral for suspected cancer. 2005 www.nice.org.uk/CG027.

12 National Institute for Health and Clinical Excellence. The recognition and initial management of ovarian cancer. 2011. www.nice.org.uk/CG122.

Cite this as: BMJ 2012:344:d7628

(c) BMJ Publishing Group Ltd 2012 